\title{
Temozolomide resistance in glioblastoma cells occurs partly through epidermal growth factor receptor- mediated induction of connexin 43
}

\author{
JL Munoz ${ }^{1,2}$, V Rodriguez-Cruz ${ }^{2}$, SJ Greco ${ }^{2}$, SH Ramkissoon ${ }^{3,4}$, KL Ligon $^{3,4}$ and P Rameshwar ${ }^{\star, 2}$
}

Glioblastoma Multiforme (GBM) is an aggressive adult primary brain tumor with poor prognosis. GBM patients develop resistance to the frontline chemotherapy, temozolomide (TMZ). As the connexins (Cx) have been shown to have a complex role in GBM, we investigated the role of $\mathrm{Cx} 43$ in TMZ resistance. Cx43 was increased in the TMZ-resistant low passage and cell lines. This correlated with the data in The Cancer Genome Atlas. Cx43 knockdown, reporter gene assays, chromatin immunoprecipitation assay, real-time PCR and western blots verified a role for $\mathrm{Cx} 43$ in TMZ resistance. This occurred by TMZ-resistant GBM cells being able to activate epidermal growth factor receptor (EGFR). In turn, EGFR activated the JNK-ERK1/2-AP-1 axis to induce Cx43. The increased $\mathrm{Cx} 43$ was functional as indicated by gap junctional intercellular communication among the resistant GBM cells. Cell therapy could be a potential method to deliver drugs, such as anti-EGF to tumor cells. Similar strategies could be used to reverse the expression of Cx43 to sensitize GBM cells to TMZ. The studies showed the potential for targeting EGF in immune therapy. These agents can be used in conjunction with stem cell therapy to treat GBM.

Cell Death and Disease (2014) 5, e1145; doi:10.1038/cddis.2014.111; published online 27 March 2014

Subject Category: Immunity

Glioblastoma Multiforme (GBM), the most common adult primary brain tumor, has poor prognosis with $<3 \%$ survival after 5 years of diagnosis. ${ }^{1}$ Currently, anti-neoplastic treatment combines chemotherapy, temozolomide ( $T M Z)$, radiotherapy and resectional surgery. ${ }^{2} \mathrm{TMZ}$ is an alkylating agent that induces apoptosis through DNA strand breaks is considered as the frontline chemotherapeutic agent for GBM. ${ }^{3}$ Despite its frontline status, GBM patients commonly exhibit resistance to $\mathrm{TMZ}$ treatment. ${ }^{4}$ Although there are several reports aimed at understanding how GBM cells resist $T M Z$, the mechanism(s) remain unclear. The reports on chemoresistance include mismatch repair of genes, cell cycle alterations and the expression of ATP-dependent drug efflux pumps. ${ }^{5}$

A number of intracellular pathways have been described in the growth and survival of GBM. These include the activation of epidermal growth factor receptor (EGFR) in GBM cells. EGFR dysfunction correlates with poor prognosis. ${ }^{6,7} \mathrm{GBM}$ patients with increased EGFR expression have enhanced activation of the EGFR variant, EGFRvIII. ${ }^{8}$ The EGFR is a member of the Erb family of receptor tyrosine kinases. Activated EGFR is a homodimeric transmembrane protein that undergoes auto-transphosphorylation through intrinsic intracellular protein-tyrosine kinase domains. ${ }^{9}$ The activation of EGFR leads to downstream signaling pathways such as phosphorylation of AKT, MAPK and JNK. ${ }^{10}$
Chemoresistance of GBM cells can occur by intercellular communication through gap junction (GJIC). ${ }^{11}$ GJIC is established by the interaction between two hemichannels on adjacent cell membranes. ${ }^{12}$ The hemichannels are formed by members of the connexin (Cx) protein family. GJIC facilitates the exchange of small molecules and microRNA. ${ }^{13,14} \mathrm{Cx} 43$ can exert both tumor suppressor and oncogenic functions. ${ }^{15}$ Astrocytes and astrocytomas express high levels of Cx43. ${ }^{16}$ During epithelial-to-mesenchymal transition and the period of overt tumor infiltration, there is a loss of GJIC between the malignant cells. ${ }^{17}$ However, the role of $\mathrm{Cx} 43$ in the migration of GBM cells is mixed. While the literature reported on a requirement for decreased $\mathrm{C} \times 43$ for GBM cells to migrate to other sites, ${ }^{17}$ another study showed a facilitating role for $\mathrm{C} \times 43$ in GBM migration. ${ }^{18}$ Together, these two opposing reports underscored the complex role of Cx43 in the development of GBM. This study investigated the role of GJIC in the early phase of acquired chemoresistance.

Here we show an increase in Cx43 in TMZ-resistant GBM cells that formed GJIC between the resistant cells. This suggested that GJIC could be a method by which resistance is communicated among GBM cells. We demonstrated that Cx43 expression was regulated at the level of transcription in the chemoresistant GBM cells. This occurred partly through the activation of EGFR1 that activated AP-1 to increase $C x 43$ transcription.

\footnotetext{
${ }^{1}$ Rutgers-Graduate School of Biomedical Science, Newark, NJ, USA; ${ }^{2}$ Department of Medicine, Hematology/Oncology, New Jersey Medical School, Rutgers School of Biomedical Health Sciences, Newark, NJ, USA; ${ }^{3}$ Department of Pathology, Brigham and Women's Hospital, Boston Children's Hospital and Harvard Medical School, Boston, MA, USA and ${ }^{4}$ Department of Medical Oncology, Dana-Farber Cancer Institute, Boston, MA, USA

${ }^{*}$ Corresponding author: P Rameshwar, Department of Medicine, Hematology/Oncology, New Jersey Medical School, Rutgers School of Biomedical Health Sciences, 185 South Orange Avenue, MSB E-585, Newark, NJ 07103, USA. Tel: + 1973972 0625; Fax: + 1973972 8854; E-mail: rameshwa@njms.rutgers.edu

Keywords: connexin 43; glioblastoma; resistance; temozolomide EGF receptor; stem cell; immune therapy

Abbreviations: Cx, connexin; Cx43, connexin 43; EGF, epidermal growth factor; EGFR, epidermal growth factor receptor; GBM, glioblastoma; Gja1, connexin 43-deficient mice; GJIC, gap junctional intercellular communication; TCGA, The Cancer Genome Atlas; TMZ, temozolomide

Received 15.10.13; revised 07.2.14; accepted 17.2.14; Edited by Y Shi
} 


\section{Results}

Cx43 expression in TMZ-resistant GBM cells. The role of GJIC has been extensively studied in the central nervous system. ${ }^{19}$ We asked whether TMZ resistance could be 'transmitted' to other cells through GJIC. We first identified the Cx type on TMZ-resistant U87 and T98G cells. Chemoresistance was established with $200 \mu \mathrm{M}$ TMZ for $72 \mathrm{~h}^{20}$ The viable cells were studied for $C x$ expression by real-time PCR and western blot. We selected Cx26, Cx32 and $C \times 43$ because of their expression on neurons, Schwann cells and astrocytes, respectively. ${ }^{19}$ Cx43 mRNA was increased by four- to six-fold and its protein was increased by three- to four-fold; Cx26 mRNA was minimally increased and Cx32 mRNA was undetectable $(C T>35)$ (Figures 1a and $b$ ). Similar findings were noted for the corresponding proteins (Figure 2b). In summary, Cx43 expression was significantly increased in the TMZ-resistant GBM cells.

As commercial U87 and T98G were passaged for many generations, we asked whether $C x$ was similarly expressed in primary GBM cells, low-passage GBM cells from patients with naive (BT145) and recurring (resistant) (BT164) GBM. Realtime PCR for Cx26, Cx32 and Cx43 cDNA indicated significant $(P<0.05)$ increases in BT164 (resistant) as compared with BT145 (naive) (Figure 1c). Western blot indicated a significant increase for $\mathrm{Cx} 43$ in the resistant BT164 cells but reduced Cx26 and undetectable Cx32 (Figure 1d).

We next analyzed the data in The Cancer Genome Atlas (TCGA) to determine whether $\mathrm{C} \times 43$ is increased in a large number of GBM tissues. The TCGA project has data analyzed using Agilent Gene Chip with $>500$ primary GBM tissues. Comparative analyses for $C_{x} 43$ expression were performed with the 522 available gene chip data, showed $C x 43$ expression with a Z-score of 2.7 and a S.D. of 1.3. This indicated an average of 2.7-fold increase in $C x 43$ expression in the GBM samples as compared with control gene expression. Similar analyses for Cx26 and Cx32 showed no significant $(P>0.05)$ change as compared with control (Figure 1e). Taken together, TMZ-resistant GBM cells, including the low-passaged cell lines, combined with the data in the TCGA gene array database, which indicated that $C \times 43$ was increased in GBM.

Sensitization of $C x 43$ knockdown GBM cells to TMZ. The studies supported a molecular role for Cx43 in TMZ resistance GBM cells (Figure 1). Functional studies investigated the sensitivity of GJA1 (Cx43) knockdown GBM cells. The specificity was studied with non-targeted siRNA (negative control). Real-time PCR and western blot verified the efficiency of Cx43 knockdown (Figures $2 a$ and b, Supplementary Figure S1). GBM cells were established as resistance to TMZ by treating with $200 \mu \mathrm{M} \mathrm{TMZ}$. At $72 \mathrm{~h}$, the cells were analyzed with a LHD release assay for cell death. As expected, only the resistant cells survived TMZ treatment in the untransfected cells, shown as $\sim 35 \%$ (Figure 2c, untransfected). There was no significant difference $(P>0.05)$ with a non-targeting siRNA. In contrast, transfection with Cx43 siRNA caused a significant $(P<0.05)$ increase in cell death as compared with cells transfected with non-targeting
siRNA (Figure 2c). These findings indicated that $C \times 43$ was involved in the acquired resistance of TMZ-treated GBM cells.

TMZ activated the $5^{\prime}$ regulatory region of GJA1 ( $\left.C_{x} 43\right)$ in GBM cells. Figures 1 and 2 supported the involvement of Cx43 in TMZ resistance. We asked if this occurs by induced Cx43. We used a reporter gene system with four different fragments of the $5^{\prime}$ regulatory region of $C \times 43$. U87 and T98G were transfected with the reporter gene vectors containing $250,350,400$ and 1400 bp upstream of the translational start site. ${ }^{21}$ Controls were transfected with vector alone. After $24 \mathrm{~h}$, the transfectants were treated with $200 \mu \mathrm{M}$ TMZ or vehicle. At $72 \mathrm{~h}$, whole-cell extracts from the viable cells were analyzed for luciferase activity. The results, presented fold change over control, indicated a significant $(P<0.05)$ increase in luciferase in the TMZ-resistant cells as compared with vehicle treatment (Figure 3a). As each fragment contained the first 250 bp of the Cx43 gene, we concluded that this fragment was important for the activation of the reporter gene during TMZ treatment.

TMZ activated AP-1. The 250-bp fragment within the $5^{\prime}$ regulatory region of GJA1 (Cx43) was identified as the active region in the TMZ-resistant cell lines (Figure 3a). To understand this further, we scanned the region for transcriptional factor binding site. We selected a potential AP-1binding site (Figure 3b) because this site has been shown to induce $C \times 43$ in other tissues. ${ }^{22}$ We transfected the GBM cells with a reporter gene that was under the control of six AP-1 tandem repeats and then treated the transfectants with $200 \mu \mathrm{M}$ TMZ. At 24,48 and $72 \mathrm{~h}$, we analyzed cell extracts for luciferase activity. In both cell lines, luciferase levels were significantly $(P<0.05)$ increased at $48 \mathrm{~h}$, whereas this consistency was not seen at the 24-h time point (Figure 3c). The results indicated that TMZ treatment led to the activation of AP-1 in the GBM cells and this occurred after $48 \mathrm{~h}$ TMZ treatment. Based on these results, we proposed that AP-1 was activated about $24 \mathrm{~h}$ before TMZ resistance. ${ }^{20}$

Interaction of AP-1 with endogenous GJA1 (Cx43). AP-1 seemed to have a role in $C x 43$ expression (Figure 3 ). As we did not mutate the AP-1-interacting sites, the studies could not definitively state that this region was responsible for the increase in $C \times 43$ transcription. We however used different methods to link the pathways in the activation of AP-1 in TMZ-resistant GBM cells.

AP-1 is a heterodimeric transcription factor composed of c-Fos and c-Jun proteins. In addition, c-Jun members can also homodimerize. ${ }^{23}$ We therefore asked which subunits were activated in the TMZ-treated U87 and T98G cells. We treated the cells for $72 \mathrm{~h}$ with $200 \mu \mathrm{M} \mathrm{TMZ}$ and then performed intracellular labeling for phospho-c-Jun and -Fos. Flow cytometric analyses indicated increased phospho-c-Jun in both cell lines and minimum phospho-c-Fos (Figures $4 a$ and b, Supplementary Figure S2).

We next asked whether AP-1 interacted with endogenous GJA1 (Cx43) in TMZ-resistant GBM using chromatin immunoprecipitation (ChIP) assay with anti-c-Jun and PCR with 
a

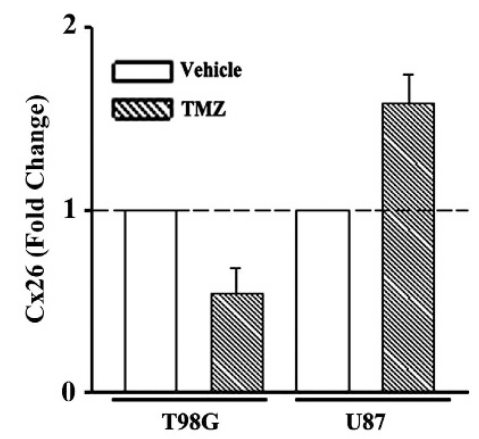

b

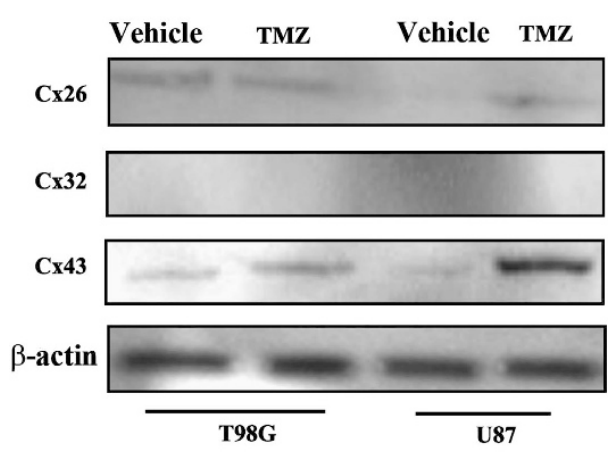

c

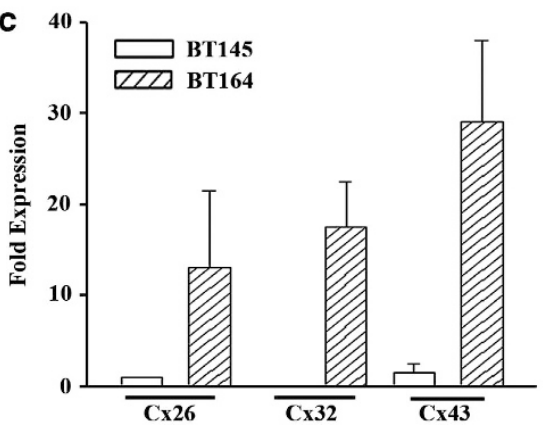

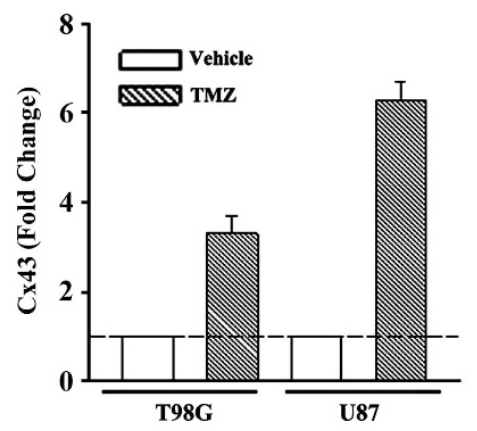

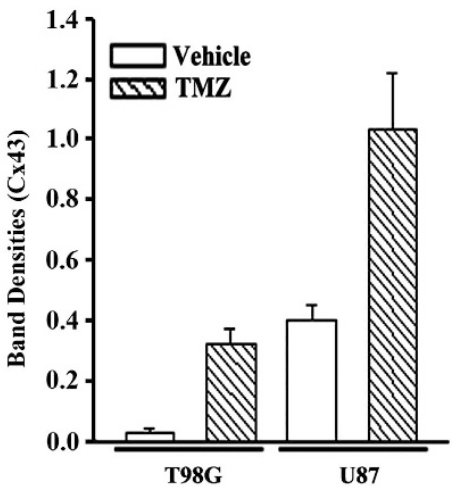

d

BT145

BT164

Cx26

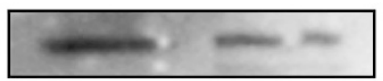

Cx32

Cx43

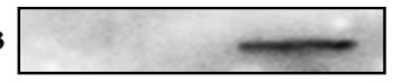

B-Actin

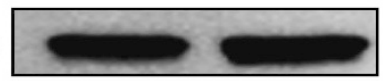

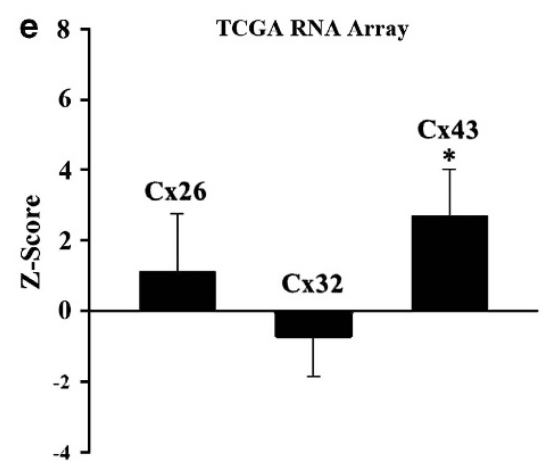

Figure 1 Increased Cx43 in TMZ-treated GBM cells. (a) U87 and T98G cells were treated with $200 \mu \mathrm{M} \mathrm{TMZ}$. After $72 \mathrm{~h}$, real-time PCR was performed with primers specific for Cx26 (left) and Cx43 (right). (b) Whole-cell extracts were prepared from U87 and T98G cells treated with TMZ for 72 h, and then analyzed by western blots for Cx26, Cx32 and Cx43 (left). The normalized band densities for Cx43 protein is shown at right. (c) RNA from low-passage cell lines from a patient with recurrent GBM (TMZ resistance BT164) and a naive patient (TMZ-sensitive BT145) were studied by real-time PCR for Cx26, Cx32 and Cx43 mRNA. The data are shown for the mean relative fold expression \pm S.D., $n=4$. (d) Whole-cell extracts from BT145 and BT164 were analyzed by western blot for $C \times 43$. (e) TCGA level 3 with $>500$ GBM tissues was used to verify the expressions of $C \times 26, C \times 32$ and $C \times 43$ expressions. The results are shown as the mean \pm S.D. for all samples within the database. ${ }^{*} P<0.05$ versus $C \times 26$ or $C \times 32$

primers spanning the AP-1 site. Analyses of the PCR product on agarose gel electrophoresis showed a bright band for TMZresistant GBMs. This indicated that $A P-1$ interacted with the CX43 gene in the TMZ-resistant GBM cells (Figure 4c).
ERK and JNK in AP-1 activation. The results shown thus far indicated that AP-1 was important for the expression of Cx43 in the TMZ-resistant GBM cells. We next asked whether the activation of AP-1 was because of upstream 


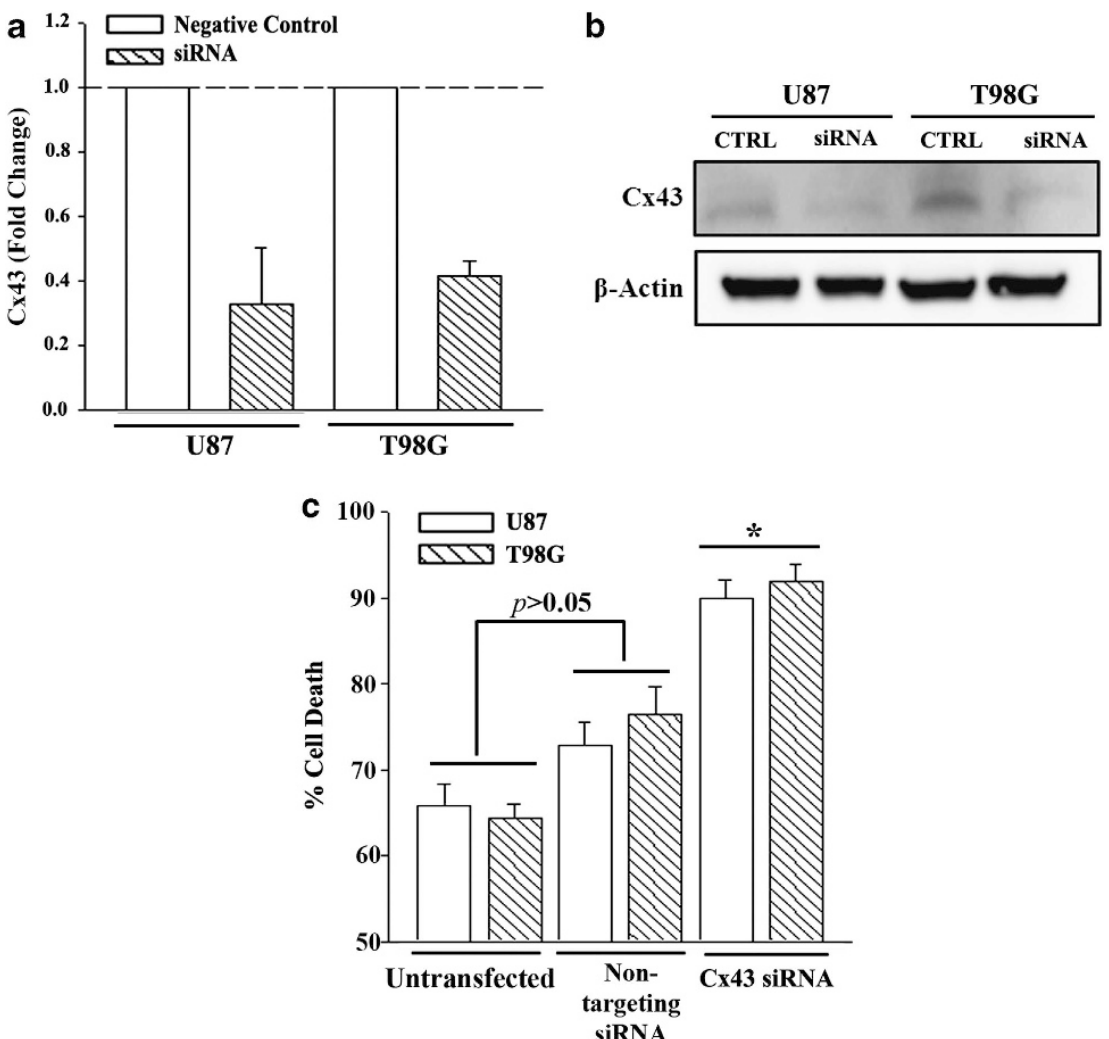

Figure 2 Cell viability of TMZ-treated Cx43 knockdown GBM cells. U87 and T98G were transfected with Cx43-targeted siRNA or a non-targeting siRNA (Negative Control, Neg Ctrl). The cells were analyzed by real-time PCR for Cx43 mRNA (a) and whole-cell extracts in western blot with anti-Cx43 (b). The knockdown cells, Neg Ctrl and untransfected cells were treated with $200 \mu \mathrm{M}$ TMZ. After $72 \mathrm{~h}$, the cells were studied for viability using the CytoTox $96 \mathrm{LDH}$ release. The results are presented as mean \pm S.D. of triplicates from each of four independent experiments $(n=12) .{ }^{*} P<0.05$ versus Neg Ctrl and untransfected cells

activation of JNK and ERK1/2. These kinases are downstream of EGFR signaling, which is commonly associated with GBM pathology. ${ }^{24}$ Thus, auto-transphosphorylation of EGFR could activate AP-1 via ERK $1 / 2$ and JNK. We performed western blot for total and phospho-ERK and JNK in untreated (time 0 ) and TMZ-treated GBM cells. Specific antibodies were used to detect unphosphorylated and the phosphorylated forms (total). The results showed time-dependent increases in phospho-ERK and -JNK (Figure 4d and Supplementary Figure S2).

EGFR1-JNK-ERK-AP-1 axis in $\mathbf{C x} 43$ expression. Studies were conducted to determine whether JNK-ERK-AP-1 axis (Figure 4d) was involved in Cx expression in the TMZ-resistant GBMs. Specific pharmacological inhibitors blocked JNK, ERK1/2 and EGFR activation. The activation of EGFR kinase was inhibited by pretreating GBM cells with Erlotinib for $24 \mathrm{~h}$. Similarly, the cells were pretreated with PD98059 to block ERK1, SP600125 to inhibit JNK and U0126 to block the effect of ERK1/2. The cells were treated with $200 \mu \mathrm{M} \mathrm{TMZ}$ for $72 \mathrm{~h}$ that were the parameters of resistance. Whole-cell extracts were analyzed by western blot for $\mathrm{Cx} 43,-32$ and -26 . If the inhibitors sensitized the GBMs to TMZ this would reduce the number of GBMs. As the housekeeping $\beta$-actin was reduced, we normalized each lane of $C x$ to $\beta$-actin (Figure $4 e$ ).

The lanes labeled as vehicle represent the control with the inhibitors and TMZ treatment. As expected, the bands were bright as the GBMs retained resistance (Figure 1b). In contrast, cells treated with the kinase inhibitors resulted in decreased $\mathrm{C} \times 43$ (Figure $4 \mathrm{e}$ and Supplementary Figure S2).

Increased activation of EGFR in TMZ-resistant GBM cells. EGFR signaling has been associated to $C \times 43$ expression. ${ }^{25}$ As Cx43 was increased in the TMZ-resistant GBMs (Figure 1), we asked whether this was caused by EGFR activation as a large number of GBM patients showed increased/activated EGFR. ${ }^{26}$ GBMs were treated with $200 \mu \mathrm{M}$ TMZ for $72 \mathrm{~h}$ or vehicle (untreated) and then analyzed for surface EGFR by flow cytometry. Phosphorylated EGFR was increased by two- to three-fold in the TMZresistant cells (Figure 5a, open histogram for resistant cells).

The studies strongly suggested that activated EGFR was involved in Cx43 expression (Figure 4e). We therefore performed cause-effect analyses by activating EGFR with exogenous rhEGF in sera-free media containing $200 \mu \mathrm{M} \mathrm{TMZ}$ and exogenous rhEGF $\left(\mathrm{IC}_{50}=50 \mathrm{ng} / \mathrm{ml}\right)$. After $72 \mathrm{~h}$, wholecell extracts were studied for $\mathrm{C} \times 43$ by western blot. The results showed significant $(P<0.05)$ increase in $\mathrm{Cx} 43$ protein in the presence of rhEGFR as compared with untreated/serafree GBM cells (Figure 5b, Supplementary Figure S3). The specificity of EGF was tested by blocking the effect of EGFR with FDA-approved anti-EGFR chimeric monoclonal antibody (Cetuximab) (Figure 5b, right lanes). 
a $\quad$ 87 pCx43-Luciferase Expression

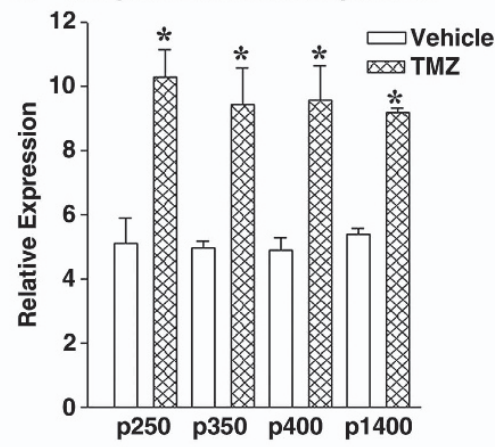

b

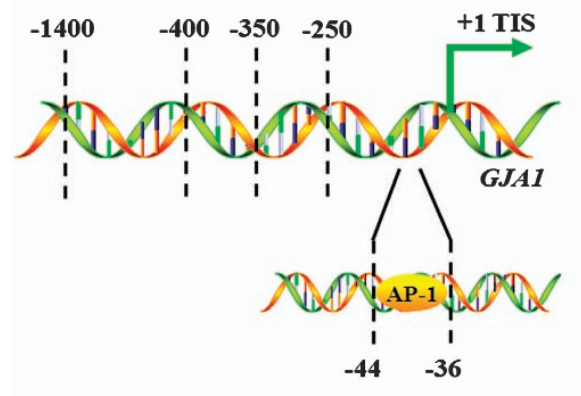

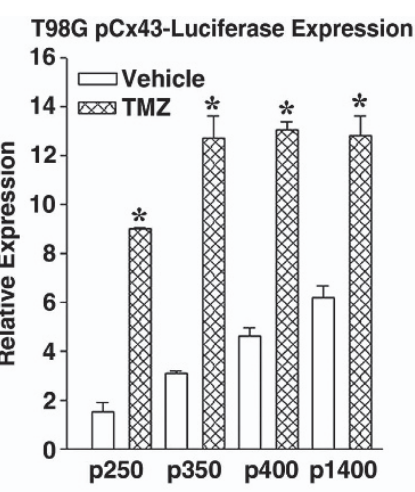

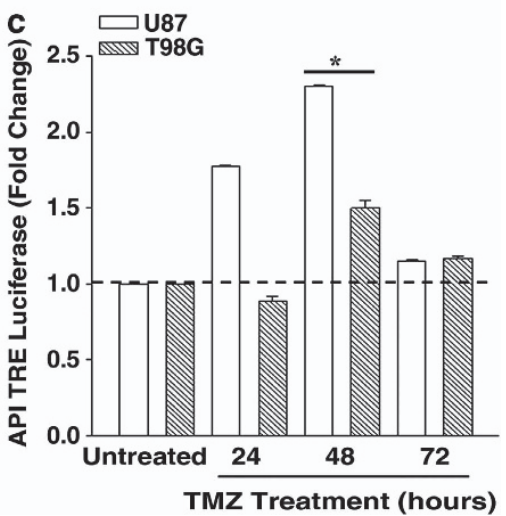

Figure 3 Effect of TMZ on the $5^{\prime}$ regulatory region of GJA1 (Cx43). T98G and U87 cells were transfected with luciferase reporter vectors with various inserts, upstream of the transcriptional initiation site of the GJA1 $5^{\prime}$ regulatory region. ${ }^{27}$ The transfectants were treated with vehicle or $200 \mu \mathrm{M} \mathrm{TMZ}$ for $72 \mathrm{~h}$. Whole-cell lysates from the viable cells were analyzed for luciferase activity. The relative expression in luciferase activities are presented as the mean \pm S.D. The data represent triplicates from four independent experiments, $n=12$ (a). Shown is the AP-1 site within the $5^{\prime}$ regulatory region Cx43 (GJA1) (b). GBM cell lines were transfected with a reporter vector containing AP-1 Tandem Repeats. The transfectants were treated with $200 \mu \mathrm{M} \mathrm{TMZ}$. After $72 \mathrm{~h}$, whole-cell extracts were studied for luciferase levels. The results are presented as mean \pm S.D. of triplicates in four independent experiments, $n=12$ (c). ${ }^{*} P<0.05$ versus vehicle

The use of Cetuximab in sera-free media indicated that EGFR is involved in Cx43 expression (Figure 5b). We therefore repeated the studies in which TMZ-resistant GBM cells were cultured in sera-containing media. The specificity for EGFR was studied with different amounts of Cetuximab. We did not use Erlotinib to block the kinase activity of EGFR but selected Cetuximab because it competed with EGF. The EGF can be present in the sera or produced by the resistant GBM cells. Thus, Cetuximab will prevent autocrine and paracrine stimulation of EGFR.

As expected, in the absence of Cetuximab (experimental point 0 ), the bands for $\mathrm{Cx} 43$ was bright, which is consistent for the TMZ-resistant cells (Figures $1 \mathrm{~b}$ and $4 \mathrm{e}$ ). However, in the presence of Cetuximab, the bands for Cx43 were decreased as compared with parallel cultures without Cetuximab (Figure 5c, 0 -EGFR; Supplementary Figure S3). Interestingly, the decrease in the bands also correlated with the disappearance of the lower bands, which are generally indicative of phosphorylated Cx43. ${ }^{27}$ The results with Cetuximab were specific because similar studies with an unrelated chimeric monoclonal anti-IgE (FDA approved, Omalizumab) did not change the bands for Cx43 (Figure 5c, left panels and Supplementary Figure S3). Together, the studies showed a role for EGFR signaling in the expression of $\mathrm{Cx} 43$ in the TMZ-resistant GBM cells.
Dye transfer between GBM cells. As the resistant GBM cells expressed $C x 43$, we asked whether the Cx43 was functional with respect to the formation of GJIC between the resistance GBM cells. Resistant GBM cells were established by treating the cells with $200 \mu \mathrm{M} \mathrm{TMZ}$ for $72 \mathrm{~h}$ as described. ${ }^{20}$ The resistant GBM cells were cocultured at 1:1 ratio of unlabeled and CMTMR (Texas Red)-labeled. Negative controls are represented as unlabeled GBM cells and positive controls as labeled GBM cells alone. After $72 \mathrm{~h}$, dye transfer was assessed by fluorescence microscopy and flow cytometry. As compared with the initial $50 \%$ labeled cells by $72 \mathrm{~h},>90 \%$ of the cells contained the CMTMR dye. This indicated that the dye was transferred from one cell to the other (Figure 6, Supplementary Figure S4). Parallel studies with 1-octanol prevented the dye transfer, indicating specificity of GJIC. In four different experiments, the transfer of dye was $>80 \%$ efficiency between the TMZ-resistant GBM cells. Representative transfer shows $83.2 \%$ for U87 and 98.8\% for T98G (Figure 6a).

\section{Discussion}

This study reported on increased expression of $C x 43$ in TMZ resistance GBM cell lines and low-passage cells (Figures 1 and 2). TMZ induced $C X 43$ transcription by an increase in EGFR that activated MAPK-AP-1 (Figures 3-6). The findings 

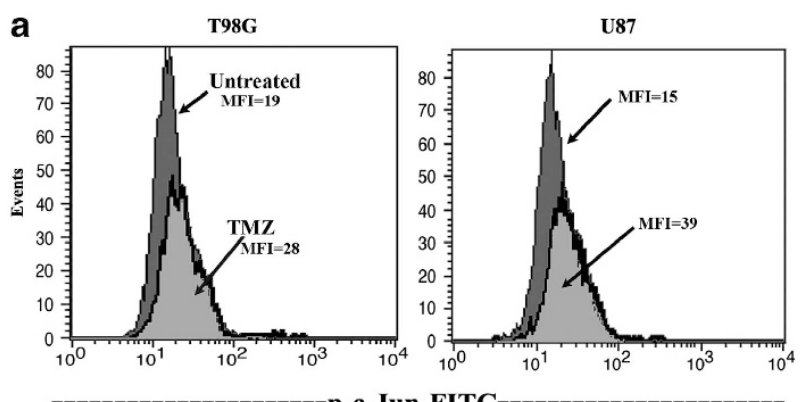

b

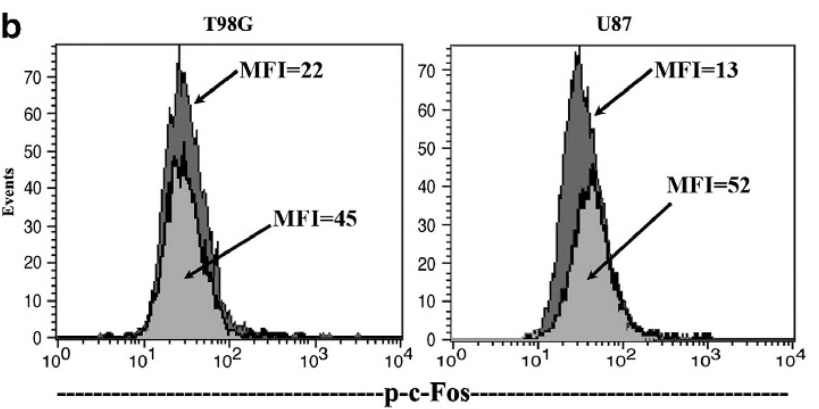

C

T98G

U87
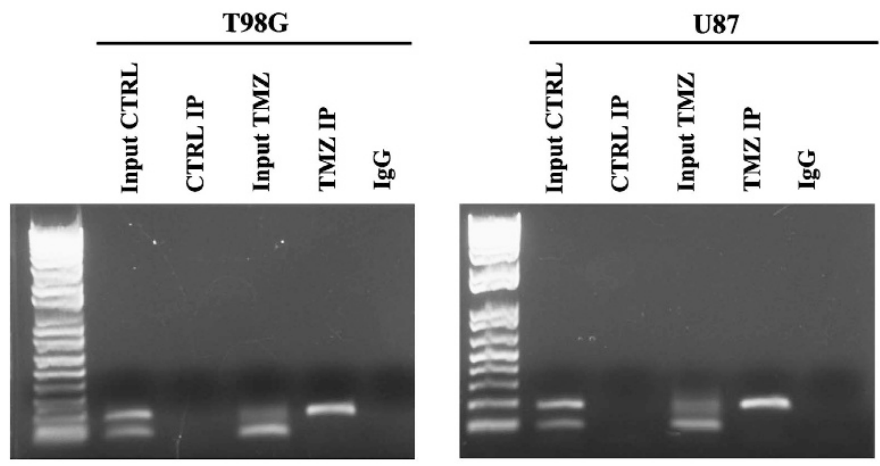

d

U87

T98G
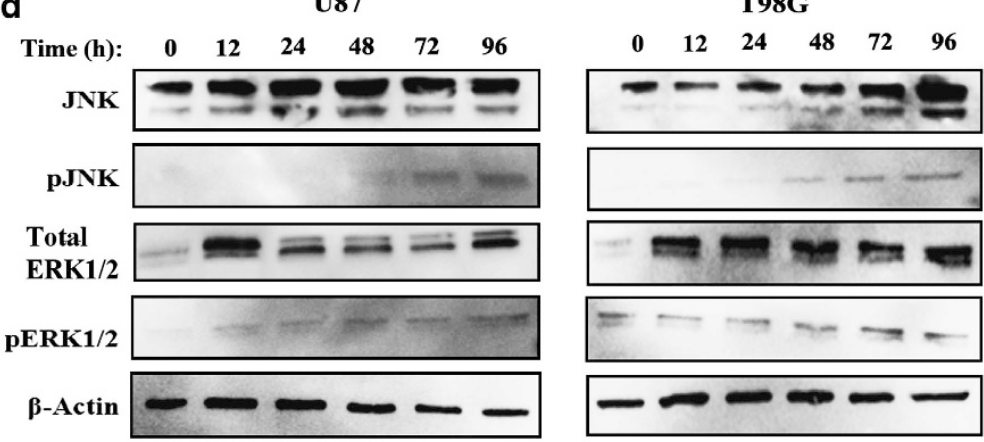

e

U87

T98G
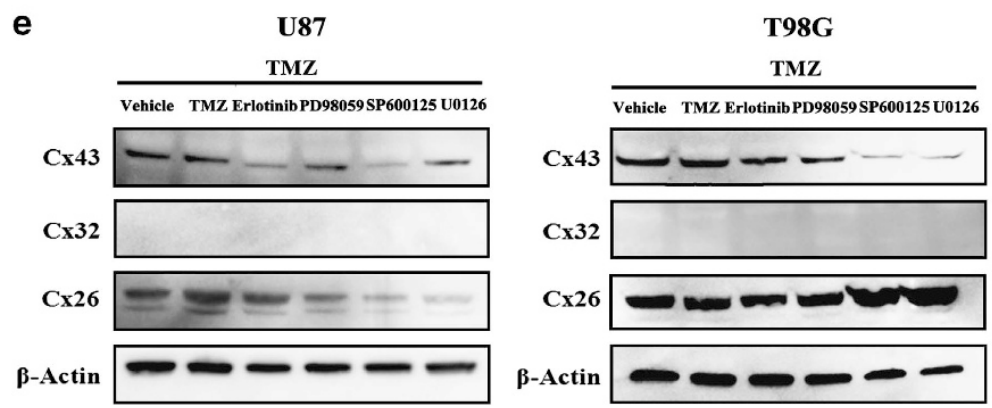

Figure 4 EGFR-mediated activation of AP-1 in TMZ-treated GBM cells. (b) U87 and T98G cells were treated with $200 \mu \mathrm{M} \mathrm{TMZ}$. After $72 \mathrm{~h}$, the viable cells were analyzed by flow cytometry for phospho (p)-c-Jun (a) and p-c-Fos activation. The dark histograms represent untreated cells and the gray histograms, TMZ treatment. The MFI of each histogram is depicted with an arrow. (c) ChIP analyses were performed for AP-1 binding to endogenous Cx43 using TMZ-resistant GBM cells. The complex was precipitated with anti-c-Jun. Shown are the PCR of the precipitated gDNA with primers spanning the AP-1 site. (d) Western blots were performed for the upstream activators of AP-1 activation, pERK and p-JNK using whole-cell extracts from untreated (Time 0) and timeline treatment of U87 and T98G with $200 \mu \mathrm{M} \mathrm{TMZ}$. The timeline studies occurred at $12 \mathrm{~h}$ intervals up to $96 \mathrm{~h}$. (e) GBM cells were pretreated different pharmacological agents along the EGFR signaling pathway. After $24 \mathrm{~h}$, the cells were washed and then exposed to $200 \mu \mathrm{M}$ TMZ for $72 \mathrm{~h}$. Whole-cell extracts were analyzed by western blots for Cx26, Cx32 and Cx43

were supported by the data in TCGA (Figure 1e). We have analyzed the chemoresistance to TMZ in long-term- $(>3$ months) and short-term (72h)-treated GBM cells (unpublished data). The treatment led to $\sim 40 \%$ cell death in both the short- and long-term exposure to TMZ; hence the 72-h time period was selected to induce $T M Z$ resistance.

The ChIP assay combined with pharmacological inhibitors supported a role for AP-1 in $C_{x} 43$ induction. The present 

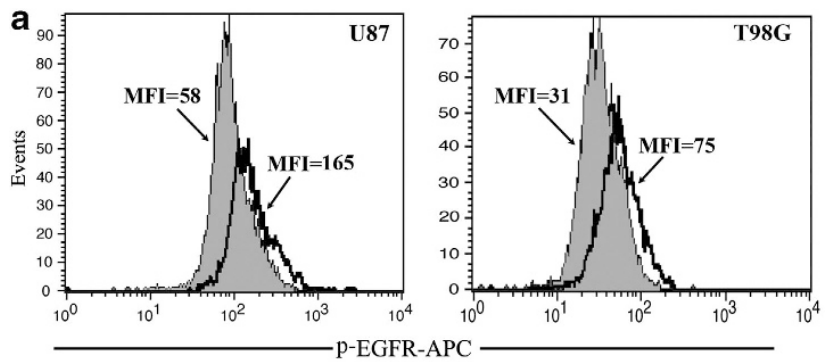

b
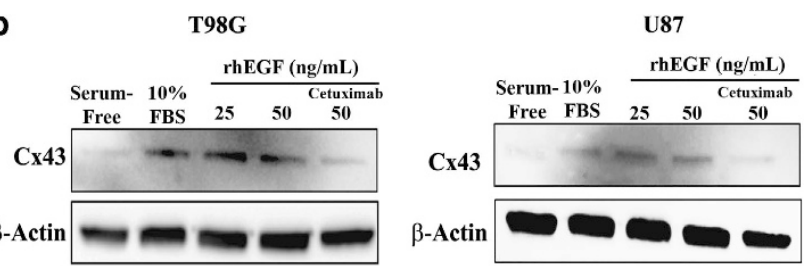

C

T98G

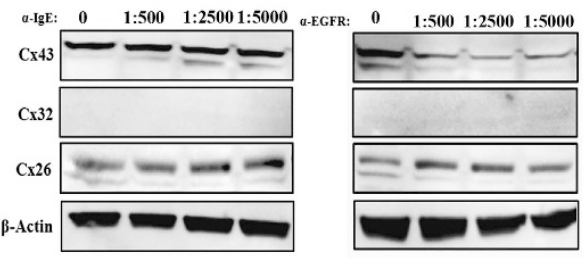

U87

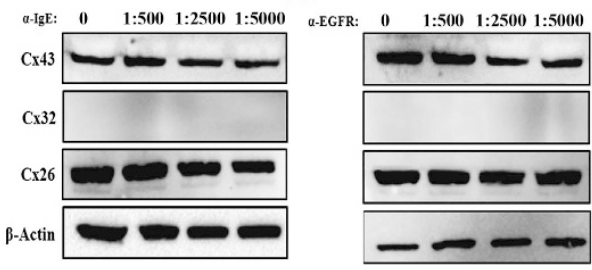

Figure 5 Effects of EGFR signaling on Cx43 expression. (a) Flow cytometry was performed for cell surface expression for EGFR with untreated and TMZresistant GBM cells. Resistance was established with $200 \mu \mathrm{M}$ TMZ for $72 \mathrm{~h}$. The solid histogram represents untreated cells and the open histogram represents treated cells. The MFI of each histogram is shown with an arrow. (b) GBM cells were treated in sera-free media with 25 and $50 \mathrm{ng} / \mathrm{ml}$ of rhEGF. In parallel, the $50 \mathrm{ng} / \mathrm{m}$ point also contained Cetuximab at 1/500 dilution. In parallel, the cells were stimulated with $10 \% \mathrm{FBS}$ alone and with serum-free medium alone. At $24 \mathrm{~h}$, wholecell lysates were analyzed by western blots for Cx43. (c) TMZ-resistant U87 and T98G cells were established with $200 \mu \mathrm{M} \mathrm{TMZ}$, in the presence or absence of Cetuximab $(\alpha-$ EGFR) or Omalizumab ( $\alpha-$ IGE). Each antibody was used at the following final dilutions: $1: 500,1: 2500$ and $1: 5000$. After $72 \mathrm{~h}$, whole-cell lysates were analyzed by western blot for $\mathrm{C} \times 26, \mathrm{C} \times 32$ and $\mathrm{C} \times 43$

report did not use the reporter gene constructs with mutant AP-1 site. Such studies are planned to fully understand how increased $\mathrm{C} \times 43$ causes chemoresistance in GBM cells. TMZmediated increase in $C \times 43$ transcription in the GBM cells would allow the formation of GJIC for exchange of molecules across gap junction (Figures $6 a$ and $b$ ). We reported on the transfer of miRNAs from chemoresistant GBM cells through exosomes. ${ }^{20}$ As miRNAs can be passed from one cell to another through GJIC, ${ }^{14}$ our findings could be another method by which resistant GBM cells survive TMZ treatment.

The resistance of GBM to TMZ with tumor relapse within months is important to understand the mechanism of resistance. ${ }^{28}$ This study identified potentially new methods to combine with current treatments for GBM. We showed how TMZ could cause GJIC for the passage of small molecules $(<2 \mathrm{kDa})$ such as miRNA. ${ }^{29}$ In the CNS, astrocytes abundantly express gap junctions for cellular maintenance and regulation. ${ }^{30} \mathrm{Cx} 43$ is the main contributor of GJIC formation in astrocytes. ${ }^{16}$ Astrocytomas commonly overexpress the same gap junction proteins as shown here for GBM, which is World Health Organization grade IV astrocytomas. ${ }^{31}$ The identification of Cx43 as key in the resistance of GBM to TMZ is in line with the argument that this could be important for the migration of GBM. ${ }^{18}$

The systematic approach used in this study showed a role for Cx43 in the resistance of GBM to TMZ. The significance of our findings in future studies was demonstrated in our final set of studies in which we showed functional GJIC between GBM cells by fluorescent dye transfer (Figure 6). The importance of the gap junctional protein in TMZ resistance was underscored in studies in which we knocked down GJA1 and demonstrated an increase in cell death to TMZ (Figure 2). Together, these experiments supported a role for $\mathrm{C} \times 43$ as a protector against TMZ treatment.

We analyzed the region within the GJA1 that showed sensitivity to TMZ using a reporter gene system. ${ }^{27}$ These studies indicated that TMZ can activate the region of GJA1 close to the transcriptional start site (Figure 3 ). The region contained an AP-1 site $(+44 /+50)$, which was shown to be functional with regard to $C x 43$ expression. ${ }^{22}$ Indeed, we showed that the AP-1 subunit, c-Jun, was phosphorylated after TMZ exposure (Figure 4d). This correlated with the ability of AP-1 to bind endogenous GJA1, as indicated by the ChIP assay (Figure 4c).

Overall, the findings showed a role for AP-1 in the induction of MDR1 in resistant GBM cells. Thus, it is expected that AP-1 transcription should be increased before full resistance, which occurs with $72 \mathrm{~h}$ TMZ treatment. Indeed, timeline studies using a reporter gene under the control of AP-1 repeats showed an increase in AP-1 activity at $48 \mathrm{~h}$ of TMZ treatment (Figure $3 \mathrm{c}$ ). This correlated with an increase in the phosphorylated form of AP-1 subunit at $72 \mathrm{~h}$ (Figure 4a). The phosphorylation of c-Jun is downstream of activated ERK $1 / 2$ and JNK (Figure 4d). These activated proteins were involved in $C \times 43$ expression (Figure 4e). As ERK $1 / 2$ and JNK can be activated by the EGFR, we focused on the involvement of EGFR on TMZ resistance of GBM cells. This data indicated an increase of functional EGFR expression, resulting in an increase in Cx43 expression (Figure 5). These findings suggested that EGFR signaling could be important even in cases where the receptor was not shown to be motivated. As a monoclonal antibody to EGFR was approved as Cetuximab by the FDA, the findings show promise for the treatment of GBM. These findings underscore the importance of taking another review on the pathogenesis of EGFR in the biology of GBM. In our ongoing studies, we have performed in vivo studies in which we were able to reverse TMZ resistance with an inhibitor of EGFR kinase. The findings will be the subject of a separate paper.

Data derived from $>500$ GBM tissues in TCGA supported the in vitro findings on increased $\mathrm{C} \times 43$ GBM cells as compared with $\mathrm{C} \times 26$ and $\mathrm{C} \times 32$ (Figure 1e). The findings were validated with two low-passage cell lines derived from an 
a
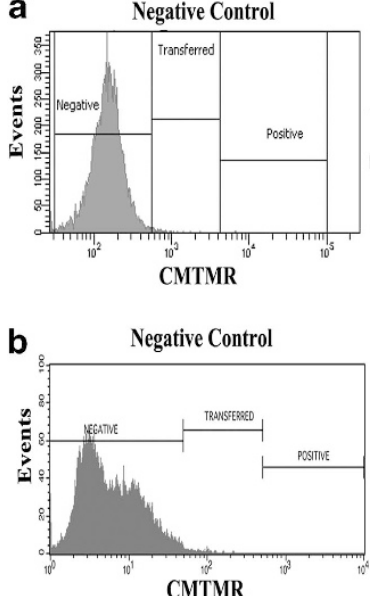

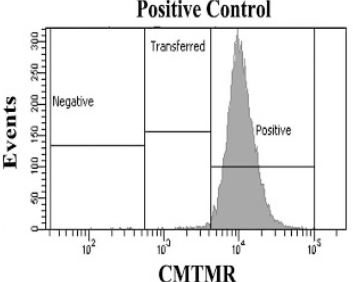

CMTMR

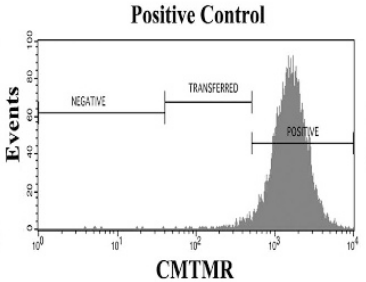

CMTMR

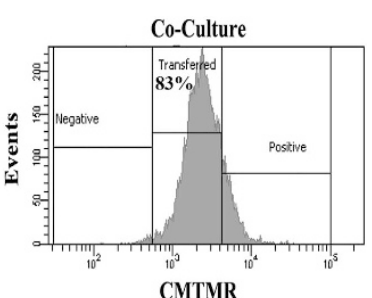

CMTMR
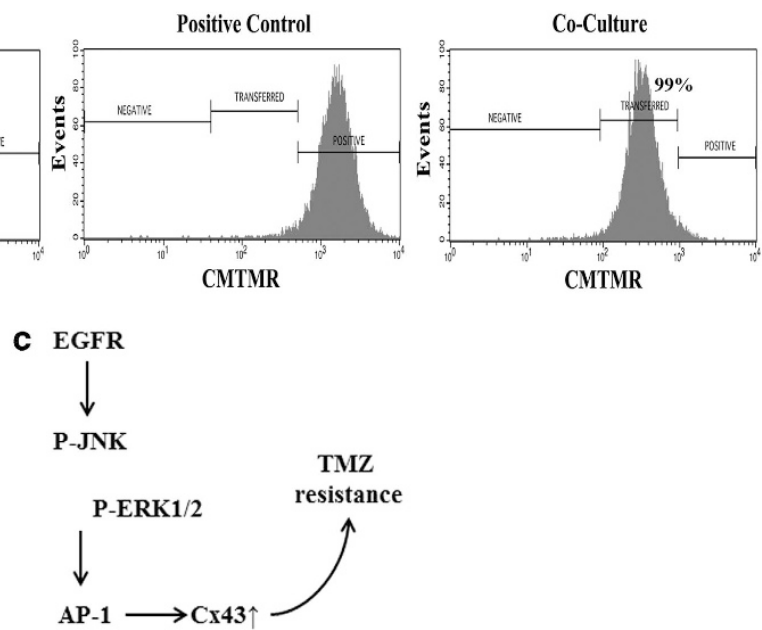

Figure 6 GBM cells form functional gap junctions. (a) Ten ${ }^{5}$ unlabeled U87 or T98G cells were cocultured for $72 \mathrm{~h}$ with ten ${ }^{5}$ CMTMR-labeled (Cell Tracker Orange) cells (center). Dye transfer was evaluated by flow cytometry. (b) A diagram shows TMZ-resistant GBM cells with activated EGFR, which phosphorylated JNK and ERK1/2 to activate AP-1 for Cx43 expression

untreated patient (BT145) and TMZ-resistant (BT164) GBM patient. More importantly, the two low-passage cell lines supported the expression of Cx43 on TMZ-resistant cells. As BT145 was never treated, it is assumed that this cell line was TMZ sensitive. Although the mRNA for the three Cxs were increased in the resistant line, only $\mathrm{C} x 43$ protein was increased in the resistant BT164 cells and the resistant established GBM cell lines (Figure 1).

Figure $6 c$ summarizes the main findings in this study. We showed EGFR signaling as essential for the increase in Cx43 in the TMZ-resistant GBM cells. The resistant GBM cells expressed higher level of EGFR (Figure 5a). However, it was unclear whether the increase was caused by TMZ. This question requires a separate study to understand how TMZ induces EGFR and how to discriminate between unphosphorylated and phosphorylated EGFR. As we demonstrated GJIC in the chemoresistant GBM cells, the findings indicated that EGFR signaling could be significant for intercellular communication and might explain how the resistance of one cell may be spread to other similar cells. The initial studies with immortalized cell lines were supported with other sources of GBM: array databases with patients' tissues and with lowpassaged cell lines from GBM. The use of pharmacological methods to decrease $\mathrm{C} \times 43$ was encouraging, as this strategy could be explored for new methods to treat GBMs. Direct targeting of $\mathrm{Cx} 43$ might be toxic as neural cells express high level of $\mathrm{Cx} 43 .{ }^{32}$ On the other hand, this study opens the 'door' to research that could lead to the identification of molecules that are exchanged between the resistant GBM cells. Their identity could be new drug targets. We showed that mesenchymal stem cells can be used to deliver anti-miRNA to resistant GBM cells. ${ }^{20}$ Similar methods could be used once future studies identified the molecules that are exchanged through GJIC. The proposed method with stem cells have been used to transfer prodrug to brain tumors. ${ }^{33}$

\section{Materials and Methods}

Cell lines. U87 and T98G World Health Organization grade IV GBM cells were purchased from American Type Culture Collection (Manassas, VA, USA) and then expanded as per manufacturer's instructions.

BT145 (primary GBM) and BT164 (recurrent GBM) glioma cell lines were derived from surgical resection material acquired from patients undergoing surgery at the Brigham and Women's Hospital on an Institutional Review Board approved protocol as part of the DF/BWCC Living Tissue Bank. Briefly, tumor resection samples were mechanically dissociated, and tumorspheres were established and propagated in Human NeuroCult NS-A Basal media (StemCell Technologies, Vancouver, BC, Canada) supplemented with EGF, FGF $\beta$ and heparin sulfate.

Reagents and antibodies. All tissue culture media were purchased from Gibco (Grand Island, NY, USA), and fetal calf serum was purchased from Hyclone Laboratories (Logan, UT, USA). Recombinant human EGF was obtained from R\&D Systems (Minneapolis, MN, USA). For fluorescent imaging, CellTracker Orange CMTMR [5-(and-6)-(4-chloromethyl) benzoyl)amino)tetramethylrhodamine] was purchased from Invitrogen (Carlsbad, CA, USA).

Mouse anti-Cx43, anti-Cx32 and anti-Cx26 were purchased from Invitrogen and, mouse anti- $\beta$-actin mAb, HRP-anti-rabbit and HRP-anti-mouse IgG from Cell Signaling Technology (Billerica, CA, USA). EGFR blocking humanized antibody (Cetuximab) and humanized lgE (Omalizumab) were kind gifts from Roche (Nutley, NJ, USA).

EGFR signaling inhibition was achieved using the following inhibitors: Erlotinib $\left(10 \mu \mathrm{M}, \mathrm{IC}_{50}=5-20 \mu \mathrm{M}\right)$, SP600125 $\left(25 \mu \mathrm{M}, \mathrm{IC}_{50}=10-50 \mu \mathrm{M}\right)$, PD98059 $(5 \mu \mathrm{M}$, $\left.\mathrm{IC}_{50}=5-10 \mu \mathrm{M}\right)$ and $\left.\mathrm{U} 012670 \mu \mathrm{M}, \mathrm{IC}_{50}=50-70 \mu \mathrm{M}\right)$. SP600125, PD98059 and U0126 were purchased from Sigma-Aldrich (St. Louis, MO, USA) and Erlotinib was purchased from Santa Cruz Biotechnology (Santa Cruz, CA, USA). 
Vectors. The GJA1 (Cx43) reporter gene (luciferase) contained inserts within the $5^{\prime}$ regulatory region. The reporter gene vectors were kindly provided by Dr. M Kandouz, Wayne State University School of Medicine (Detroit, MI, USA). The vectors were previously described. ${ }^{21}$ The pGL3 luciferase backbone vector (Promega, Madison, WI, USA) served as vector control. AP-1 activity was evaluated with pGL4.44[luc2P/AP-1 RE/Hygro, which is a 6x Tandem Repeat Element of AP-1, upstream of luciferase (Promega).

Dye transfer. U87 or T98G cells $\left(5 \times 10^{5}\right)$ were seeded in six-well plates and then incubated with $2.5 \mu \mathrm{M}$ of prewarmed CellTracker Orange CMTMR [5-(and-6)(((4-chloromethyl) benzoyl)amino) tetramethylrhodamine] (Life Technologies - Molecular Probes, Grand Island, NY, USA). After the CMTMR enters the cells, it reacts with the intracellular components and cannot exit the cells. Thus to ensure specificity, GJIC studies are done in the presence of an inhibitor, 1-octanol. The cells were incubated for $45 \mathrm{~min}$ at $37^{\circ} \mathrm{C}$ in a $\mathrm{CO}_{2}$ incubator. After this, the media were removed and the cells were washed three times with PBS. Fresh culture media were added to the washed cells. The plates were incubated for $6 \mathrm{~h}$ and then subjected to a second wash with PBS. After this, the labeled cells were cocultured with $5 \times 10^{5}$ unlabeled cells. After $72 \mathrm{~h}$, the cells were trypsinized, centrifuged at $500 \times g$, resuspended in $0.5 \mathrm{ml}$ of PBS and then immediately evaluated by flow cytometry for dye transfer.

Real-time RT-PCR. RNA was extracted with Trizol reagent (Invitrogen). Reverse transcription was performed using the High Capacity CDNA Reverse Transcription Kit (Life Technologies - Applied Biosystems, Grand Island, NY, USA) in accordance with the manufacturer's recommendation. Real-time PCR was performed with $200 \mathrm{ng}$ cDNA on a 7300 Real-Time PCR System (Applied Biosystems). The initial incubation was at $50^{\circ} \mathrm{C}$ for $2 \mathrm{~min}$ followed by $95^{\circ} \mathrm{C}$ for $10 \mathrm{~min}$. After this, the cycling conditions were as follows: $95^{\circ} \mathrm{C}$ for $15 \mathrm{~s}$ and $60^{\circ} \mathrm{C}$ for $60 \mathrm{~s}$, for 40 cycles. Primer sequences used in the PCR were: Cx26: (F) 5'-AGCTCTGCTCCCCTAAAG-3' (R) 5'-TGTGTCCTCTGTGGAACC-3'; CX32: (F) 5'-GGCATTCTACTGCCATTG-3', (R) 5'-TGGGGTGGAAACTAGGAT-3'; CX43: (F) 5'-TCGGGTTAAGGGAAAGAG-3', (R) 5'-GCTCACTTGCTTGCTTGT-3'; $\beta$-actin: (F) $5^{\prime}$-TGCCCTGAGGCACTCTTC-3' and (R) $5^{\prime}$-GTGCCACCAGGGCAGT GATCT-3'. Primers were purchased from Sigma (St. Louis, MO, USA). The relative expression was calculated using 2( - Delta Delta $\mathrm{C}(\mathrm{T}))$, as previously described. ${ }^{14}$

Western blot. GBM cells were treated with $200 \mu \mathrm{M}$ TMZ or with vehicle. After $72 \mathrm{~h}$, whole-cell extracts were isolated with M-PER Mammalian Protein Extraction Reagent (Thermo Scientific, Rockford, IL, USA). Cell extracts (3-7 $\mu \mathrm{g}$ ) were analyzed by western blots, as previously described on 12\% SDS-PAGE gels (Bio-Rad, Hercules, CA, USA) ${ }^{27}$ Proteins were transferred to PVDF membranes (PerkinElmer, Boston, MA, USA). The membranes were incubated overnight with primary antibodies at final dilutions ranging between $1 / 500$ and $1 / 1000$. Primary antibodies were detected by incubating for $2 h$ with HRP-conjugated $\lg G$ at $1 / 2000$ final dilution. HRP activity was assayed by chemiluminescence using SuperSignal West Femto Maximum Sensitivity Substrate (Thermo Scientific). Membranes were stripped with Restore Stripping Buffer (Thermo Scientific) and then reprobed with other antibodies.

Flow cytometry. The cell surface expression for EGFR was analyzed by flow cytometry with 1/100 final dilution of anti-EGFR (Cetuximab with human Fc). This was followed by incubation with 1/500 mouse anti-human IgG (bridging antibody). A third incubation used 1/1000 FITC-conjugated goat-anti-mouse IgG. The fluorescence intensity was determined with a FACS Calibur (BD Biosciences, San Jose, CA, USA).

Cell death analyses. Lactate dehydrogenase (LDH) release assays (Promega) studied cell death. The LDH assay was based on the disruption of the cell membrane. The LDH assay was assessed at $72 \mathrm{~h}$ after TMZ treatment. ${ }^{34}$ We measured the conversion of Formazan (Red) using a Victor3V Multilabel Plate Reader (PerkinElmer; Waltham, MA, USA) with an emission filter of $490 \mathrm{~nm}$. The results were normalized to complete cell lysis. This was aided by a lysis buffer included in the LDH assay kit.

Analyses of the $5^{\prime}$ regulatory region of GJA1. The minimal promoter of the GJA1 gene was identified on the basis of the information in the NCBI database (NC_000006.11). This region was identified as $-250 \mathrm{bp}$, relative to the transcriptional initiation site, and was also supported by luciferase activity (see Results section). The 250-bp region was analyzed for potential transcriptional binding regions using Genematix platform (Munich, Germany).
ChIP assay. ChIP analysis for AP-1-interacting site on the GJA1 gene was performed using the ChIP-it Express Enzymatic Kit (Active Motif, Carlsbad, CA, USA), as described. ${ }^{35,36} \mathrm{~A}$ ChIP-validated anti-c-Jun antibody was also purchased from Active Motif. GJA1 isolation was confirmed by qPCR. Samples were isolated during the exponential phase of synthesis as determined by qPCR and run on $1 \%$ agarose gel. Although two bands were amplified from genomic input DNA, only the upper band (200 bp) was isolated by ChIP. The upper band was confirmed to be GJA1 PCR.

Transfection and reporter gene assay. U87 and T98G cells were transfected with the GJA1 reporter gene constructs, using Effectene Transfection Reagent (Qiagen, Valencia, CA, USA). After $72 \mathrm{~h}$, protein lysates were obtained using M-PER Mammalian Protein Extraction Reagent (Thermo Scientific), and quantified for protein concentration (Bio-Rad). Luciferase activity was calculated as per total protein and then presented as relative light units. The relative light units was normalized to untreated lysates.

Statistical analysis. Data were analyzed using the Student's t-test for two comparable groups (control/experimental). A $P$-value $<0.05$ was considered significant.

\section{Conflict of Interest}

The authors declare no conflict of interest.

Acknowledgements. This work was supported by the F M Kirby Foundation and this work is in partial fulfillment of JLM's PhD thesis. VR-C was supported by the Research Initiative for Science Enhancement Program at the University of Puerto Rico in Cayey (R25 GM59429). We would also like to thank Sukhwinder Singh, PhD and the Flow Cytometry and Immunology Core Laboratory at New Jersey Medical School.

1. Shahar T, Nossek E, Steinberg DM, Rozovski U, Blumenthal DT, Bokstein F et al. The impact of enrollment in clinical trials on survival of patients with glioblastoma. J Clin Neurosci 2012; 19: 1530-1534.

2. Park CK, Lee SH, Kim T, Choi S, Park SH, Heo D et al. The value of temozolomide in combination with radiotherapy during standard treatment for newly diagnosed glioblastoma. J Neurooncol 2013; 112: 277-283.

3. Zhang J, Stevens MF, Bradshaw TD. Temozolomide: mechanisms of action, repair and resistance. Curr Mol Pharmacol 2012; 5: 102-114.

4. Johannessen TCA, Bjerkvig R. Molecular mechanisms of temozolomide resistance in glioblastoma multiforme. Expert Rev Anticancer Ther 2012; 12: 635-642.

5. Happold C, Roth P, Wick W, Schmidt N, Florea AM, Silginer M et al. Distinct molecular mechanisms of acquired resistance to temozolomide in glioblastoma cells. J Neurochem 2012; 122: 444-455.

6. Joshi AD, Loilome W, Siu IM, Tyler B, Gallia GL, Riggins GJ. Evaluation of tyrosine kinase inhibitor combinations for glioblastoma Therapy. PLOS One 2012; 7: e44372.

7. Lv S, Teugels E, Sadones J, De BS, Duerinck J, Du FS et al. Correlation of EGFR, IDH1 and PTEN status with the outcome of patients with recurrent glioblastoma treated in a phase II clinical trial with the EGFR-blocking monoclonal antibody cetuximab. Int J Oncol 2012; 41: 1029-1035.

8. Del Vecchio CA, Li G, Wong AJ. Targeting EGF receptor variant III: tumor-specific peptide vaccination for malignant gliomas. Expert Rev Vaccines 2012; 11: 133-144.

9. Bai J, Guo XG, Bai XP. Epidermal growth factor receptor-related DNA repair and radiationresistance regulatory mechanisms: a mini-review. Asian Pac J Cancer Prev 2012; 13: 4879-4881

10. Meng Q, Xia Y. c-Jun at the crossroad of the signaling network. Protein Cell 2011; 2: 889-898.

11. Gielen PR, Aftab Q, Ma N, Chen VC, Hong X, Lozinsky S et al. Connexin43 confers Temozolomide resistance in human glioma cells by modulating the mitochondrial apoptosis pathway. Neuropharmacol 2013; 75: 539-548.

12. Bennett MVL, Garre JM, Orellana JA, Bukauskas FF, Nedergaard M, Giaume C et al. Connexin and pannexin hemichannels in inflammatory responses of glia and neurons. Brain Res 2012; 1487: 3-15.

13. Sin WC, Crespin S, Mesnil M. Opposing roles of connexin43 in glioma progression. Biochim Biophys Acta 2012; 1818: 2058-2067.

14. Lim PK, Bliss SA, Patel SA, Taborga M, Dave MA, Gregory LA et al. Gap junction-mediated import of microRNA from bone marrow stromal cells can elicit cell cycle quiescence in breast cancer cells. Cancer Res 2011; 71: 1550-1560.

15. Warn-Cramer BJ, Lau AF. Regulation of gap junctions by tyrosine protein kinases. Biochim Biophys Acta 2004; 1662: 81-95. 
16. Caltabiano R, Torrisi A, Condorelli D, Albanese V, Lanzafame S. High levels of connexin 43 mRNA in high grade astrocytomas. Study of 32 cases with in situ hybridization. Acta Histochemica 2010; 112: 529-535.

17. Crespin S, Bechberger J, Mesnil M, Naus CC, Sin WC. The carboxy-terminal tail of connexin43 gap junction protein is sufficient to mediate cytoskeleton changes in human glioma cells. J Cell Biochem 2010; 110: 589-597.

18. Naus CC, Laird DW. Implications and challenges of connexin connections to cancer. Nat Rev Cancer 2010; 10: 435-441.

19. Rouach N, Avignone E, Meme W, Koulakoff A, Venance L, Blomstrand F et al. Gap junctions and connexin expression in the normal and pathological central nervous system. Biol Cell 2002; 94: 457-475.

20. Munoz JL, Bliss SA, Greco SJ, Ramkissoon SH, Ligon KL, Rameshwar P. Delivery of functional anti-miR- 9 by mesenchymal stem cell-derived exosomes to glioblastoma multiforme cells conferred chemosensitivity. Mol Ther Nucleic Acids 2013; 2: e126.

21. Carystinos GD, Kandouz M, Alaoui-Jamali MA, Batist G. Unexpected induction of the human connexin 43 promoter by the Ras signaling pathway is mediated by a novel putative promoter sequence. Mol Pharmacol 2003; 63: 821-831.

22. Echetebu CO, Ali M, Izban MG, MacKay L, Garfield RE. Localization of regulatory protein binding sites in the proximal region of human myometrial connexin 43 gene. Mol Human Reprod 1999; 5: 757-766.

23. Shaulian E. AP-1-The Jun proteins: Oncogenes or tumor suppressors in disguise? Cell Signalling 2010; 22: 894-899.

24. Peterziel H, Muller J, Danner A, Barbus S, Liu HK, Radlwimmer B et al. Expression of podoplanin in human astrocytic brain tumors is controlled by the PI3K-AKT-AP-1 signaling pathway and promoter methylation. Neuro Oncol 2012; 14: 426-439.

25. Park JH, Lee MY, Heo JS, Han HJ. A potential role of connexin 43 in epidermal growth factor-induced proliferation of mouse embryonic stem cells: Involvement of $\mathrm{Ca} 2+/ \mathrm{PKC}$, p44/42 and p38 MAPKs pathways. Cell Prolif 2008; 41: 786-802.

26. Hegi ME, Rajakannu $P$, Weller M. Epidermal growth factor receptor: a re-emerging target in glioblastoma. Curr Opin Neurol 2012; 25: 774-779.

27. Park JM, Munoz JL, Won BW, Bliss SA, Greco SJ, Patel SA et al. Exogenous CXCL12 activates protein kinase $C$ to phosphorylate connexin 43 for gap junctional intercellular communication among confluent breast cancer cells. Cancer Lett 2013; 331: 84-91.
28. Chamberlain MC. Temozolomide: therapeutic limitations in the treatment of adult highgrade gliomas. Expert Rev Neurother 2010; 10: 1537-1544.

29. Batra N, Kar R, Jiang JX. Gap junctions and hemichannels in signal transmission function and development of bone. Biochimica Biophysica Acta 2012; 1818: 1909-1918.

30. Theis M, Giaume C. Connexin-based intercellular communication and astrocyte heterogeneity. Brain Res 2012; 1487: 88-98.

31. Abrams CK, Scherer SS. Gap junctions in inherited human disorders of the central nervous system. Biochimica Biophysica Acta 2012; 1818: 2030-2047.

32. Marquez-Rosado L, Solan JL, Dunn CA, Norris RP, Lampe PD. Connexin43 phosphorylation in brain, cardiac, endothelial and epithelial tissues. Biochim Biophys Acta 2012; 1818: 1985-1992.

33. Lee HK, Finniss S, Cazacu S, Bucris E, Ziv-Av A, Xiang C et al. Mesenchymal stem cells deliver synthetic microRNA mimics to glioma cells and glioma stem cells and inhibit their cell migration and self-renewal. Oncotarget 2013; 4: 346-361.

34. Ulasov IV, Nandi S, Dey M, Sonabend AM, Lesniak MS. Inhibition of Sonic hedgehog and Notch pathways enhances sensitivity of CD133( +) glioma stem cells to temozolomide therapy. Mol Med 2011; 17: 103-112.

35. Patel N, Klassert TE, Greco SJ, Patel SA, Munoz JL, Reddy BY et al. Developmental regulation of TAC1 in peptidergic-induced human mesenchymal stem cells: implication for spinal cord injury in zebrafish. Stem Cells Dev 2012; 21: 308-320.

36. Greco SJ, Smirnov SV, Murthy RG, Rameshwar P. Synergy between the RE-1 silencer of transcription and NFkappaB in the repression of the neurotransmitter gene TAC1 in human mesenchymal stem cells. J Biol Chem 2007; 282: 30039-30050.

(c) Cell Death and Disease is an open-access journal
published by Nature Publishing Group. This work is
licensed under a Creative Commons Attribution 3.0 Unported License.
To view a copy of this license, visit http://creativecommons.org/
licenses/by/3.0/

Supplementary Information accompanies this paper on Cell Death and Disease website (http://www.nature.com/cddis) 